\title{
Criminologie
}

\section{Les enfants autochtones en protection de la jeunesse au Québec : leur réalité comparée à celle des autres enfants}

\author{
Alexandra Breton, Sarah Dufour et Chantal Lavergne
}

Volume 45, numéro 2, automne 2012

URI : https://id.erudit.org/iderudit/1013724ar

DOI : https://doi.org/10.7202/1013724ar

Aller au sommaire du numéro

\section{Éditeur(s)}

Les Presses de l’Université de Montréal

ISSN

0316-0041 (imprimé)

1492-1367 (numérique)

Découvrir la revue

Citer cet article

Breton, A., Dufour, S. \& Lavergne, C. (2012). Les enfants autochtones en protection de la jeunesse au Québec : leur réalité comparée à celle des autres enfants. Criminologie, 45(2), 157-185. https://doi.org/10.7202/1013724ar
Résumé de l'article

Les recherches canadiennes et internationales s'entendent sur la surreprésentation des enfants autochtones en protection de l'enfance. La présente étude ajoute aux connaissances sur les mauvais traitements et la réponse des services de protection de la jeunesse aux enfants autochtones québécois. Elle poursuit d'abord l'objectif d'analyser la surreprésentation des enfants autochtones à trois étapes de prise de décision en protection de la jeunesse. Les deux groupes d'enfants, autochtones et non autochtones, seront comparés en fonction d'un ensemble de caractéristiques, tant sur le plan personnel que sur celui de leur famille et de leurs parents. Il sera aussi question des signalements dont ils font l'objet et des services qui leur sont rendus. Les prédicteurs du placement des enfants desservis par la protection de la jeunesse sont enfin vérifiés, en portant une attention particulière à l'importance du statut autochtone dans cette prédiction. Les résultats révèlent une augmentation de la surreprésentation des enfants autochtones d'une étape à l'autre des services de protection. Ces enfants ont plus souvent des conditions de vie difficiles et sont exposés à davantage de facteurs de risque que les autres enfants. Le statut autochtone est important dans la prédiction du placement, même après l'ajout d'un ensemble de caractéristiques pouvant contribuer à la prédiction. Les implications pour la recherche et la pratique sont discutées. 


\title{
Les enfants autochtones en protection de la jeunesse au Québec: leur réalité comparée à celle des autres enfants
}

\author{
Alexandra Breton \\ Spécialiste en activités cliniques \\ Centre jeunesse de Montréal-Institut universitaire \\ alexandra.breton@cjm-iu.qc.ca \\ Sarah Dufour \\ Professeure agrégée \\ École de psychoéducation, Université de Montréal \\ sarah.dufour@umontreal.ca \\ Chantal Lavergne \\ Chercheure \\ Centre jeunesse de Montréal-Institut universitaire \\ chantal.lavergne@cjm-iu.qc.ca
}

\begin{abstract}
RÉSUMÉ - Les recherches canadiennes et internationales s'entendent sur la surreprésentation des enfants autochtones en protection de l'enfance. La présente étude ajoute aux connaissances sur les mauvais traitements et la réponse des services de protection de la jeunesse aux enfants autochtones québécois. Elle poursuit d'abord l'objectif d'analyser la surreprésentation des enfants autochtones à trois étapes de prise de décision en protection de la jeunesse. Les deux groupes d'enfants, autochtones et non autochtones, seront comparés en fonction d'un ensemble de caractéristiques, tant sur le plan personnel que sur celui de leur famille et de leurs parents. Il sera aussi question des signalements dont ils font l'objet et des services qui leur sont rendus. Les prédicteurs du placement des enfants desservis par la protection de la jeunesse sont enfin vérifiés, en portant une attention particulière à l'importance du statut autochtone dans cette prédiction. Les résultats révèlent une augmentation de la surreprésentation des enfants autochtones d'une étape à l'autre des services de protection. Ces enfants ont plus souvent des conditions de vie difficiles et sont exposés à davantage de facteurs de risque que les autres enfants. Le statut autochtone est important dans la prédiction du placement, même après l'ajout d'un ensemble de caractéristiques pouvant contribuer à la prédiction. Les implications pour la recherche et la pratique sont discutées.
\end{abstract}

MOTS-CLÉS - Enfants autochtones, mauvais traitements, protection de la jeunesse, surreprésentation, placement. 


\section{Introduction ${ }^{1}$}

La présente recherche s'intéresse à la situation des enfants autochtones dans le système de protection de la jeunesse québécois. On considère comme autochtone «toute personne ayant déclaré appartenir à au moins un groupe autochtone, c'est-à-dire Indien de l'Amérique du Nord, Métis ou Inuit, et/ou toute personne ayant déclaré être un Indien des traités ou un Indien, inscrit tel que défini dans la Loi sur les Indiens $d u$ Canada, et/ou toute personne ayant déclaré appartenir à une bande indienne et/ ou à une Première Nation [sic]» (Statistique Canada, 2006a). Le Québec comptait 108425 Autochtones en 2006, représentant 1,5\% de la population. De ces Autochtones, les enfants et jeunes de 0 à 19 ans représentaient 37105 individus, soit 34,2\% de la population autochtone vivant au Québec (Statistiques Canada, 2006b). La population autochtone québécoise n'est pas homogène; elle est composée de 11 nations, réparties en 14 villages inuits et 41 communautés amérindiennes (Gaudreault, 2009).

La discrimination systémique sert de cadre conceptuel à la présente recherche. Ce concept englobe à la fois la discrimination directe (une personne soumise à un traitement différent sur un motif de discrimination prohibé) et indirecte (pratique, en apparence neutre, qui a des effets préjudiciables sur les membres de groupes visés par la Charte des droits et libertés de la personne du Québec, dont les Autochtones) (Eid et al., 2011). Il s'intéresse aux interrelations dynamiques entre diverses pratiques, au sein des institutions et entre elles, qui ont des effets préjudiciables, voulus ou non, sur les groupes visés par la Charte (Bosset, 2005 ; Gouvernement du Québec, 2006; Eid et al., 2011). Comme les lois canadienne et québécoise garantissent maintenant l'égalité entre les individus, plusieurs croient que le rapport de domination imposé aux Autochtones relève du passé (Commission sur le racisme systémique dans le système de justice pénale en l'Ontario, 1995 ; Cotton, 2008). Sans prétendre que la discrimination à l'égard des peuples autochtones du Canada soit encore aujourd'hui consciente ou délibérée, il reste que les relations entre Autochtones et non-Autochtones sont souvent problématiques, teintées de rapports de pouvoir et où les premiers sont souvent désavantagés socialement et politiquement (Salee, 2005). De

1. Cette recherche a été financée par le Conseil de recherches en sciences humaines du Canada. Une bourse de maîtrise offerte à la première auteure par le Centre jeunesse de Montréal-Institut universitaire a aussi facilité sa réalisation. 
nombreuses études, menées par des organisations gouvernementales canadiennes et de spécialistes des politiques sociales, concluent que les Autochtones continuent d'être victimes de discrimination de la part d'individus et d'organisations (Commission sur le racisme de l'Ontario, 1995 ; Eid et al., 2011). La Commission des droits de la personne et des droits de la jeunesse du Québec (Eid et al., 2011) soulignait récemment que la société doit porter un regard critique sur ses institutions, dont le Directeur de la protection de la jeunesse, afin de s'assurer qu'elles ne produisent pas d'effet discriminatoire systémique.

La surreprésentation des enfants autochtones dans les services de protection

Différentes recherches ont mis en évidence la surreprésentation des enfants autochtones dans les services de protection de l'enfance en Amérique du Nord. La surreprésentation réfère au fait que la place relative de certains groupes culturels dans les services de protection ne reflète pas leur poids démographique dans la population (Child Welfare League of America, 2005). À l'échelle nationale aux États-Unis, les enfants autochtones sont surreprésentés aux différentes étapes des services de protection: ils sont deux fois plus nombreux que ce qui avait été anticipé aux signalements et aux situations fondées et de deux à trois fois plus nombreux que leur proportion dans la population générale à l'étape du placement (Hill, 2007). Au Minnesota, les enfants autochtones sont quatre à cinq fois plus à risque de faire partie des cas de mauvais traitements fondés, en comparaison de la population majoritaire de référence (Ards et al., 2003).

Au Canada, trois vastes études d'incidence des cas de mauvais traitements (CIS-1998; CIS-2003 et CIS-2008) réalisées depuis 1998 permettent d'apprécier l'importance de la surreprésentation des enfants autochtones dans les services de protection de l'enfance. Une analyse des données du CIS-1998 révèle la présence d'une grande disparité entre les enfants autochtones et les autres aux différentes étapes de prises de décision, en particulier à celle de la corroboration des faits (55\% c. $42 \%$ pour les non-Autochtones) et à celle du placement $(29 \%$ c. $12 \%$; Blackstock et al., 2004). Une autre analyse réalisée cette fois à partir du CIS-2003 montre que les enfants autochtones sont surreprésentés dans les signalements aux services de protection canadiens (18\% des signalements c. $5 \%$ dans la population; Lavergne et al., 2008). 
Enfin, les résultats du CIS-2008 indiquent que les taux d'évaluations fondées sont près de quatre fois plus importants chez les enfants autochtones en comparaison des enfants non autochtones. Au total, $22 \%$ des évaluations fondées concernent des enfants d'origine autochtone (Public Health Agency of Canada, 2010).

Si la disproportion des enfants autochtones dans le système de protection a été documentée dans le reste du Canada, des efforts restent encore à mener au Québec. À notre connaissance, seuls Tourigny, Domond, Trocmé, Sioui et Baril (2007) ont comparé des enfants autochtones et non autochtones à partir d'un échantillon représentatif des signalements faits au Directeur de la protection de la jeunesse québécoise en 1998. Selon cette étude, au regard des problématiques fondées, il existe une proportion plus grande d'enfants victimes de négligence chez les Autochtones (49\% c. $37 \%$ ) alors que leur proportion est plus faible pour la présence de troubles de comportement $(18 \%$ c. $33 \%)$. Les autres problématiques étaient présentes en proportions similaires entre les groupes.

Trois principaux éléments sont invoqués pour expliquer cette surreprésentation dans les services de protection: des actions assimilatrices passées, des conditions de vie dégradées en raison de nombreux facteurs de risque et l'existence possible de biais dans le traitement des cas d'enfants autochtones dans les services de protection de l'enfance (Trocmé et al., 2004). Durant les années 1800 et jusqu'au milieu des années 1900, les efforts d'assimilation par les gouvernements et les représentants de l'Église ont contribué à éteindre la culture autochtone des enfants. Ces derniers étaient retirés à leurs parents et à leur communauté pour être conduits dans les pensionnats; ils ne pouvaient alors plus parler leur langue ni conserver de traces de leur identité culturelle (Commission royale sur les peuples autochtones, 1996). Dans ces pensionnats, les abus physiques et sexuels étaient courants (Milloy, 1999). Le gouvernement fédéral reconnaît maintenant les injustices et les sévices subis par les Autochtones. Il s'en est excusé officiellement en 1998, puis en 2008, et a dédommagé financièrement les anciens pensionnaires (Radio-Canada, 2008). Les pratiques de protection ont aussi contribué à l'assimilation des enfants autochtones à la majorité non autochtone. Durant les années ayant suivi la période des pensionnats, les enfants ont été massivement retirés de leur milieu familial, souvent pour être relocalisés à l'extérieur de leur communauté d'origine. Les intervenants en protection de la jeunesse n'avaient pas suffisamment d'informations 
ou de ressources pour comprendre les situations de pauvreté, la perte de pouvoir d'agir ainsi que les blessures intergénérationnelles vécues dans les réserves (Blackstock et al., 2004; Sinha et al., 2011). Jusqu'à récemment, les familles non autochtones étaient favorisées lorsque les enfants autochtones faisaient l'objet d'un placement ou devaient être adoptés. Les pensionnats et les pratiques de protection sont susceptibles d'avoir contribué à l'effritement de l'identité culturelle de ces enfants autochtones, qui sont aujourd'hui devenus parents, voire grands-parents. Il est plausible que la difficulté de nombreux parents autochtones à prendre soin de leurs enfants provienne de l'absence de modèles parentaux positifs lors de leur passage dans les pensionnats (Trocmé et al., 2004).

Tout comme les enfants afro-américains, eux aussi surreprésentés dans les services de protection, les enfants autochtones canadiens et québécois connaissent des conditions de vie associées à une augmentation des risques de mauvais traitements. La surconsommation d'alcool représente un grave problème au sein de ces communautés (MacLaurin et al., 2008). Les femmes autochtones sont par ailleurs à plus haut risque d'être victimes de violence conjugale que l'ensemble des femmes canadiennes $(25 \%$ c. $8 \%)$ (Jiwani, 2000). Sachant qu'il existe une forte cooccurrence entre la violence conjugale et la violence à l'égard des enfants (Miller-Perrin et Perrin, 2007), cette réalité est préoccupante. Lavergne et al. (2008) ont comparé les caractéristiques d'enfants autochtones, blancs, noirs, asiatiques ou issus d'autres minorités visibles signalés aux services de protection canadiens (excluant le Québec). Leurs résultats montrent que la négligence et les formes multiples de mauvais traitements concernent davantage les enfants autochtones, lorsque ceux-ci sont comparés aux autres groupes. Les enfants autochtones sont en outre plus nombreux à être signalés en bas âge (0-5 ans) et par des sources non professionnelles (entourage de l'enfant). Les parents d'enfants autochtones éprouvent un plus grand nombre de problèmes et les familles ont des conditions d'existence plus difficiles. Lorsqu'on les compare aux enfants non autochtones, les enfants autochtones signalés pour maltraitance vivent dans des conditions socioéconomiques plus difficiles, sont davantage signalés et vivent avec des parents qui ont plus de difficultés, particulièrement la toxicomanie et les activités criminelles. Toutefois, lorsque l'on compare les familles autochtones et non autochtones canadiennes vivant des difficultés similaires, il n'y a pas de différence en ce qui concerne les décisions 
de placement. Ces décisions semblent donc influencées par les caractéristiques de la situation plutôt que l'ethnicité (Trocmé et al., 2004). Ces conclusions tendent à infirmer l'hypothèse selon laquelle il existerait des biais dans le traitement des dossiers autochtones en protection de l'enfance. En somme, si les actions assimilatrices ont d'abord contribué à la surreprésentation des enfants autochtones en protection de l'enfance, il est possible que cette situation s'explique maintenant davantage par les conditions de vie difficiles que connaissent les familles autochtones.

\section{Caractéristiques liées aux placements des enfants autochtones}

Malgré les faits historiques relatifs aux placements des enfants autochtones évoqués plus haut et la sensibilité de cette mesure de protection pour cette clientèle, les motifs menant au placement des enfants autochtones comparativement aux enfants non autochtones sont encore mal connus.

Aux États-Unis, Donald, Bradley, Day, Critchley et Nuccio (2003) ont comparé des enfants autochtones et non autochtones placés dans l'État du Minnesota aux États-Unis en 1996; les résultats révèlent que les enfants autochtones ont trois ans de moins que les enfants non autochtones. Ils vivent dans la pauvreté dans $93 \%$ des cas, ont le plus souvent été victimes de négligence physique et grandissent surtout dans des familles monoparentales. L'usage d'alcool se révèle en outre plus prévalent chez les parents autochtones que chez les parents non autochtones. À partir d'une analyse secondaire de données nationales aux États-Unis, Carter (2009) a exploré les caractéristiques pouvant prédire le placement d'enfants autochtones et des Premières Nations d'Alaska, comparés à des enfants non autochtones. Les trois principaux prédicteurs du placement sont l'abus d'alcool chez les parents, les problèmes de santé mentale parentaux et la pauvreté économique. Les analyses descriptives ont pourtant montré que les enfants autochtones et des Premières Nations d'Alaska ne se distinguent pas significativement des enfants non autochtones quant aux problèmes d'abus d'alcool ou de pauvreté économique. L'auteur a donc soulevé la possibilité d'un racisme institutionnel pour expliquer ces résultats.

Au Canada, Trocmé et al. (2004) ont vérifié si les décisions prises par les professionnels en protection de la jeunesse étaient influencées par le statut ethnoculturel des enfants, en comparant des enfants autoch- 
tones et blancs. Leurs résultats révèlent que les risques de placement sont significativement plus élevés lorsque le signalement est corroboré, qu'il implique une famille reconstituée ou monoparentale, dans laquelle où il $\mathrm{y}$ a eu deux déménagements ou plus au cours de l'année et dont la source de revenus est du travail à temps partiel. De plus, les probabilités de placement augmentent lorsque les problèmes de comportement des enfants sont plus importants et que le parent présente un ou plusieurs des problèmes suivants: implication dans la criminalité, déficits cognitifs, problèmes de consommation d'alcool ou historique de mauvais traitements durant sa propre enfance. Les cas d'abus sexuel et d'exposition à la violence familiale sont nettement moins susceptibles de mener à un placement que les cas d'abus physique. Les auteurs concluent que les plus hauts taux de placements chez les enfants autochtones s'expliquent davantage par une combinaison de caractéristiques à risque qu'en raison de leur appartenance ethnoculturelle (Trocmé et al., 2004). Enfin, Fluke et ses collaborateurs (2010) ont vérifié les effets des facteurs cliniques (situation de l'enfant et de sa famille) et organisationnels (services de protection) dans la décision de placer un enfant dans les services de protection canadiens. Le statut autochtone de l'enfant ne contribue pas à la prédiction du placement de manière significative. Le nombre de signalements d'enfants autochtones auprès d'une organisation constitue le seul prédicteur organisationnel des décisions de placement.

\section{Questions de recherche}

La présente recherche vise à répondre aux questions suivantes. Au sein du système de protection de la jeunesse québécois, comparativement aux autres enfants, 1- Les enfants autochtones sont-ils surreprésentés aux étapes des signalements retenus, des situations fondées et des placements? 2- Présentent-ils des caractéristiques différentes des autres enfants sur les plans personnel, familial, des signalements dont ils font l'objet et des services qui leur sont rendus? 3- Quelle est la contribution relative du statut autochtone dans la décision de placement au terme de l'évaluation et de l'orientation des cas, par rapport à l'influence des autres facteurs propres à l'enfant, à la famille, aux parents, ainsi qu'aux caractéristiques du signalement et des services rendus? 


\section{Méthode}

Recrutement des centres jeunesse et sujets à l'étude

Les 11 centres jeunesse (CJ) québécois desservant une réserve ou communauté autochtone ont été invités à collaborer à la recherche ${ }^{2}$. Neuf d'entre eux ont accepté: Gaspésie/Les Îles, Québec/Institutuniversitaire, Mauricie et Centre-du-Québec, Côte-Nord, Montérégie, Outaouais, Abitibi-Témiscamingue, Lanaudière et Laurentides. Les centres jeunesse du Bas-Saint-Laurent et du Saguenay-Lac-Saint-Jean, qui desservent $12 \%$ des enfants autochtones habitant les territoires visés par l'étude, ont décliné l'invitation.

La population étudiée comprend tous les enfants de 0 à 17 ans signalés au Directeur de la protection de la jeunesse au cours de la période du 9 juillet 2007 au 8 juillet 2008. Les 16529 enfants signalés au moins une fois durant la période à l'étude sur les territoires participants ont été divisés en deux groupes selon leur appartenance culturelle: un groupe d'enfants autochtones $(n=1017)$ et un groupe d'enfants non autochtones $(\mathrm{n}=15512)$. Pour qu'un enfant soit inclus dans le groupe autochtone, lui-même ou au moins un de ses parents doit être autochtone. Un enfant est classé dans le groupe enfants non autocbtones s'il n'est pas identifié comme autochtone.

\section{Variables et sources des données}

La présente étude constitue une analyse secondaire des données cliniques et administratives extraites du système clientèle des centres jeunesse ayant accepté de participer au projet. Depuis 2003, tous les dossiers des enfants qui entrent dans les services québécois de protection de la jeunesse sont informatisés par le biais de l'outil clinique Projet intégrateur jeunesse (PIJ). Les informations contenues dans ces dossiers sont ensuite versées périodiquement dans une banque de données informationnelles (BDI) utilisable par les centres jeunesse et les chercheurs (voir Lavergne et al., 2005 pour des détails). À ce jour, chaque centre jeunesse québécois possède sa propre BDI. Il a donc été nécessaire de contacter la direction de chacun d'entre eux pour solliciter sa participation à l'étude. Les variables documentées concernent les

2. Aucun centre jeunesse ne dessert les enfants du Nunavik (nation inuite) et de la Baie-James (nation crie). 
caractéristiques des enfants, des familles, des parents, des signalements et des services rendus à l'enfant. La distribution des données manquantes a été vérifiée entre les $\mathrm{CJ}$ et entre les groupes (Autochtones c. nonAutochtones) et aucun biais n'est observé. Malgré son importance théorique et empirique, la pauvreté des familles n'a pu être incluse dans les analyses car cette information n'est pas documentée dans le système PIJ. Le calcul des taux de disproportion utilise les données du recensement canadien de 2006. Les variables ont été choisies en se basant sur les écrits scientifiques au sujet des facteurs de risque liés aux mauvais traitements et au placement (voir l'annexe I pour une définition détaillée de chacune des variables de l'étude).

\section{Analyses}

La réponse à la première question de recherche portant sur la surreprésentation se fait par le calcul de l'indice de disparité pour chacun des groupes (Autochtones/non-Autochtones). Cet indice représente la probabilité, pour un groupe, de faire l'expérience d'un événement précis, comparativement à la probabilité qu'un autre groupe fasse l'expérience du même événement (Shaw et al., 2008). La comparaison des groupes (deuxième question) est réalisée à l'aide de khi-deux et de test t. Enfin, la troisième question est vérifiée à l'aide d'une régression logistique, avec une sélection de modèles utilisant la méthode du pas à pas sur la fonction de vraisemblance. À la suite des analyses préliminaires, non présentées ici, seules les variables indépendantes significatives dans la distinction par chi carré entre les deux groupes d'enfants (autochtones/non autochtones) (placés oui/non), dans leur corrélation avec la variable dépendante (placés oui/non) et dans leur force d'association selon le test du V de Cramer sont conservées.

\section{Résultats}

Les indices de disparité présentés au tableau 1 témoignent de la surreprésentation des enfants autochtones à trois étapes de prise de décision en protection. Comparativement aux autres enfants, les enfants autochtones ont trois fois plus de probabilités de voir leur signalement retenu pour évaluation. Après évaluation, comparativement à un enfant non autochtone, il est quatre fois plus probable que la sécurité ou le développement d'un enfant autochtone soit considéré compromis. Enfin, les 


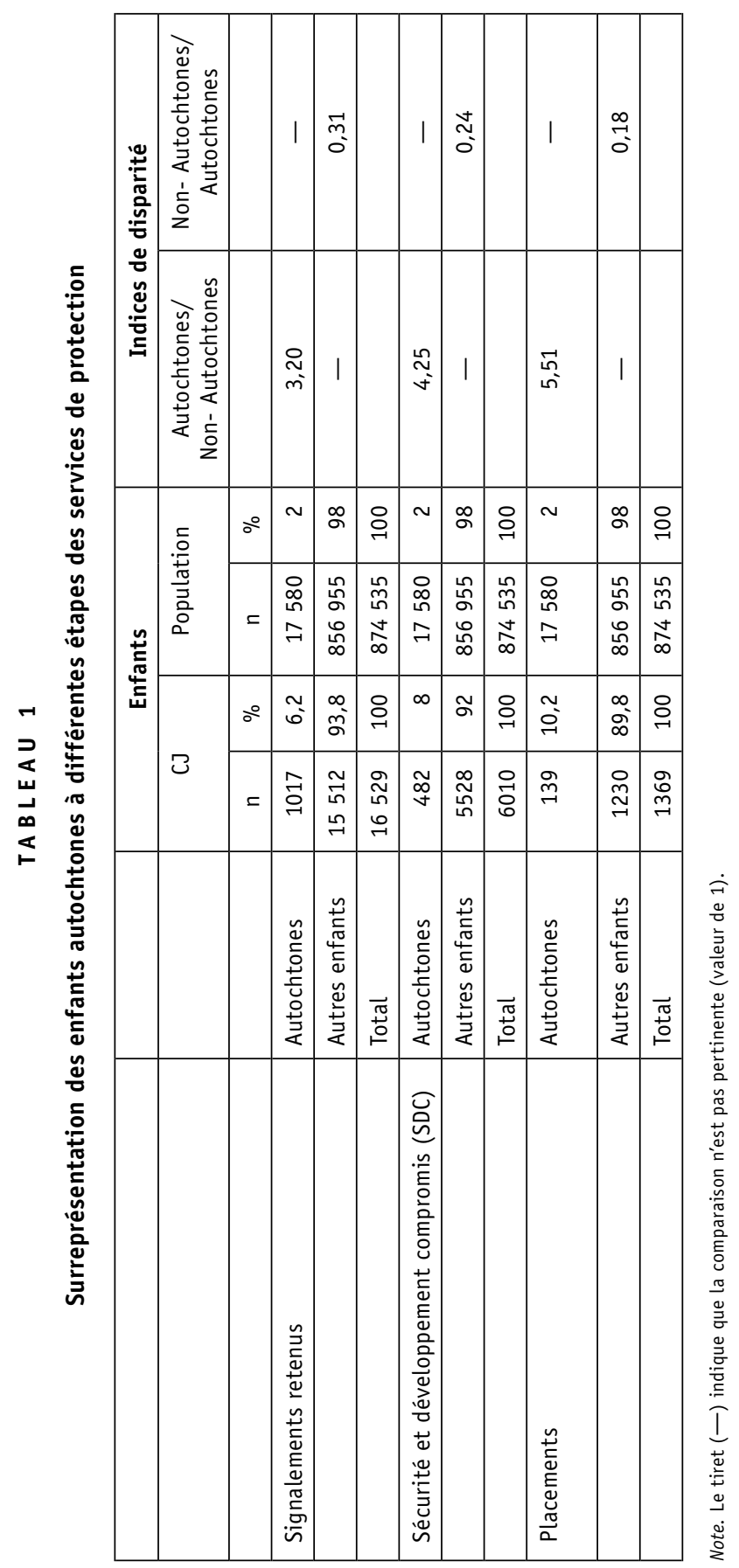


enfants autochtones sont cinq fois et demie plus susceptibles de vivre un placement que les autres. Bref, non seulement les enfants autochtones sont surreprésentés à toutes les étapes de prise de décision en protection, mais cette surreprésentation augmente à mesure qu'ils progressent dans leurs trajectoires de services.

Les tableaux 2 à 5 présentent les résultats des analyses comparatives. Au regard des caractéristiques des enfants et de leur famille, les résultats indiquent que les enfants autochtones dont le signalement est retenu sont plus jeunes que les autres enfants. Ils ont un plus grand nombre de frères et sœurs. Leurs parents éprouvent plus de problèmes que ceux des enfants non autochtones (toxicomanie, instabilité, violence conjugale, nombre moyen de problématiques), mais ils ont moins souvent un problème de santé mentale que les autres parents. Tout comme pour les enfants non autochtones, les signalements des enfants autochtones sont le plus souvent retenus pour négligence ou cooccurrence de mauvais traitements. Un signalant professionnel (employé du réseau de la santé et des services sociaux, d'école, de services de garde ou policier) est proportionnellement plus fréquent qu'un signalant non professionnel, que l'enfant soit autochtone ou non. Les enfants autochtones sont plus souvent signalés par un employé du réseau de la santé et des services sociaux que les autres enfants et moins souvent par un employé de l'école ou de leur milieu de garde. On attribue plus souvent un degré de priorité élevé (immédiat) pour l'évaluation d'une situation d'enfant

TABLEAU 2

Comparaison des caractéristiques des enfants en fonction de leur appartenance ethnoculturelle

\begin{tabular}{|l|c|c|c|c|c|c|}
\hline \multirow{2}{*}{} & \multicolumn{2}{|c|}{ Autochtones } & \multicolumn{2}{|c|}{ Non-Autochtones } & \multicolumn{2}{l|}{} \\
\cline { 2 - 7 } & $\mathrm{n}$ & $\%$ & $\mathrm{n}$ & $\%$ & $\chi^{2}$ & $p$ \\
\hline Âge & & & & & 44,05 & $<0,0005$ \\
\hline $0-5$ ans & 373 & 40,9 & 4516 & 30,5 & & \\
\hline $6-11$ ans & 271 & 29,7 & 5014 & 33,8 & & \\
\hline $12-17$ ans & 268 & 29,4 & 5289 & 35,7 & & \\
\hline Total & 912 & 100 & 14819 & 100 & & \\
\hline Sexe & & & & & 1,32 & 0,25 \\
\hline Masculin & 465 & 51 & 7265 & 49 & & \\
\hline Féminin & 447 & 49 & 7554 & 51 & & \\
\hline Total & 912 & 100 & 14819 & 100 & & \\
\hline
\end{tabular}


TA B LEA U 3

Comparaison des caractéristiques familiales en fonction de l'appartenance ethnoculturelle de l'enfant

\begin{tabular}{|l|c|c|c|c|c|c|}
\hline & \multicolumn{2}{|c|}{ Autochtones } & \multicolumn{2}{c|}{ Non-Autochtones } & \multicolumn{2}{l|}{} \\
\cline { 2 - 7 } & $\mathrm{n}$ & $\%$ & $\mathrm{n}$ & $\%$ & $\chi^{2}$ & $p$ \\
\hline $\begin{array}{l}\text { Nombre d'enfants } \\
\text { dans la fratrie }\end{array}$ & & & & & 10,56 & $<0,0005$ \\
\hline Enfant unique & 227 & 24,9 & 5570 & 37,6 & & \\
\hline Deux enfants & 188 & 20,6 & 5275 & 35,6 & & \\
\hline Trois enfants ou plus & 497 & 54,5 & 3974 & 26,8 & & \\
\hline Total & 912 & 100 & 14819 & 100 & & \\
\hline Violence familiale & & & & & 0,21 & 0,65 \\
\hline Non & 793 & 90,5 & 13293 & 90,1 & & \\
\hline Oui & 83 & 9,5 & 1468 & 9,9 & & \\
\hline Total & 876 & 100 & 14761 & 100 & & \\
\hline
\end{tabular}

autochtone, à la suite de laquelle sa sécurité ou son développement est plus souvent jugé compromis. Enfin, les enfants autochtones ont plus fréquemment été signalés une fois ou plus avant l'étude (récurrence) et sont plus souvent placés au terme de l'évaluation et de l'orientation du cas que les enfants non autochtones.

Enfin, le tableau 6 présente les variables dans l'équation de la régression logistique au modèle final, c'est-à-dire une fois l'ensemble des caractéristiques pertinentes incluses dans le modèle de prédiction. Le khi-deux du modèle est $\left[\chi^{2}(19, \mathrm{n}=5392)=637,24, \mathrm{p}<0,0001\right]$. L'indice $R^{2}$ de Nagelkerke s'élève à 0,169 . Cette faible part de variance expliquée à partir du modèle (environ $17 \%$ ) suggère l'existence de variables non incluses dans le modèle qui pourraient fournir une explication plus complète des conditions qui augmentent les probabilités de placement (p. ex. : variables socio-économiques). Au regard des caractéristiques de l'enfant, lorsqu'il est identifié comme étant d'origine autochtone, il a près de deux fois plus de probabilités d'être placé qu'un autre enfant. Comparativement aux enfants d'âge préscolaire (0-5 ans), les adolescents (12-17 ans) présentent une plus grande probabilité d'être placés. Les enfants uniques présentent aussi une plus grande probabilité d'être placés que les enfants avec fratrie. Quant aux caractéristiques parentales, lorsqu'il y a présence de toxicomanie dans le foyer d'un enfant, celui-ci a plus de probabilité de se retrouver placé; la situation s'inverse en présence de violence conjugale. Les 
TABLEA U 4

Comparaison des caractéristiques parentales en fonction de l'appartenance ethnoculturelle de l'enfant

\begin{tabular}{|c|c|c|c|c|c|c|}
\hline & \multicolumn{2}{|c|}{ Autochtones } & \multicolumn{2}{|c|}{ Non-Autochtones } & \multirow[b]{2}{*}{$\chi^{2}$} & \multirow[b]{2}{*}{$p$} \\
\hline & $n$ & $\%$ & $n$ & $\%$ & & \\
\hline Toxicomanie & & & & & 35,76 & $<0,0005$ \\
\hline Non & 369 & 42,1 & 11558 & 78,3 & & \\
\hline Oui & 507 & 57,9 & 3203 & 21,7 & & \\
\hline Total & 876 & 100 & 14761 & 100 & & \\
\hline Instabilité & & & & & 12,18 & $<0,0005$ \\
\hline Non & 485 & 55,4 & 12012 & 81,4 & & \\
\hline Oui & 391 & 44,6 & 2749 & 18,6 & & \\
\hline Total & 876 & 100 & 14761 & 100 & & \\
\hline Problème de santé mentale & & & & & 33,09 & $<0,0005$ \\
\hline Non & 845 & 96,5 & 13397 & 90,8 & & \\
\hline Oui & 31 & 3,5 & 1364 & 9,2 & & \\
\hline Total & 876 & 100 & 14761 & 100 & & \\
\hline Violence conjugale & & & & & 48,05 & $<0,0005$ \\
\hline Non & 684 & 78,1 & 12761 & 86,5 & & \\
\hline Oui & 192 & 21,9 & 2000 & 13,5 & & \\
\hline Total & 876 & 100 & 14761 & 100 & & \\
\hline Conflits de couple & & & & & 0,19 & 0,66 \\
\hline Non & 778 & 88,8 & 13038 & 88,3 & & \\
\hline Oui & 98 & 11,2 & 1723 & 11,7 & & \\
\hline Total & 876 & 100 & 14761 & 100 & & \\
\hline Antécédents de violence & & & & & 1,61 & 0,20 \\
\hline Non & 863 & 98,5 & 14449 & 97,9 & & \\
\hline Oui & 13 & 1,5 & 312 & 2,1 & & \\
\hline \multirow[t]{2}{*}{ Total } & 876 & 100 & 14761 & 100 & & \\
\hline & $\mathrm{m}$ & é.t. & $\mathrm{m}$. & é.t. & $\mathrm{t}$ & $p$ \\
\hline $\begin{array}{l}\text { Nombre de problématiques } \\
\text { parentales }\end{array}$ & 1,56 & 1,41 & 0,93 & 1,25 & 14,39 & $<0,0005$ \\
\hline
\end{tabular}

probabilités de placement varient aussi en fonction des types de problématiques de protection: les enfants signalés pour troubles de comportement ou cooccurrence de problématiques ont une probabilité plus importante d'être placés que les enfants signalés pour négligence, 
TA B LEA U 5

Comparaison des caractéristiques du signalement et des services rendus en fonction de l'appartenance ethnoculturelle de l'enfant

\begin{tabular}{|c|c|c|c|c|c|c|}
\hline & \multicolumn{2}{|c|}{ Autochtones } & \multicolumn{2}{|c|}{ Non-Autochtones } & \multirow[b]{2}{*}{$\chi^{2}$} & \multirow[b]{2}{*}{$p$} \\
\hline & $n$ & $\%$ & $\mathrm{n}$ & $\%$ & & \\
\hline \multicolumn{5}{|l|}{ Problématique de protection } & 2,31 & $<0,0005$ \\
\hline Négligence et abandon & 364 & 39,9 & 3928 & 26,5 & & \\
\hline Abus physique & 48 & 5,3 & 2105 & 14,2 & & \\
\hline Abus sexuel & 72 & 7,9 & 1580 & 10,7 & & \\
\hline Troubles de comportement & 65 & 7,1 & 1465 & 9,9 & & \\
\hline $\begin{array}{l}\text { Mauvais traitement } \\
\text { psychologique }\end{array}$ & 29 & 3,2 & 1148 & 7,7 & & \\
\hline Deux ou trois problématiques & 334 & 36,6 & 4591 & 31 & & \\
\hline Total & 912 & 100 & 14817 & 100 & & \\
\hline \multicolumn{5}{|l|}{ Signalant } & 28,67 & $<0,0005$ \\
\hline Non-professionnel & 347 & 39,2 & 5021 & 35,5 & & \\
\hline Employé SSSS & 276 & 31,2 & 3813 & 26,9 & & \\
\hline Employé école, milieu de garde & 128 & 14,4 & 3043 & 21,5 & & \\
\hline Police & 135 & 15,2 & 2277 & 16,1 & & \\
\hline Total & 886 & 100 & 14154 & 100 & & \\
\hline \multicolumn{5}{|c|}{ Degré de priorité accordé à la demande } & 20,39 & $<0,0005$ \\
\hline Immédiat & 181 & 20,4 & 2247 & 15,9 & & \\
\hline 24 heures & 104 & 11,7 & 2267 & 16,0 & & \\
\hline 4 jours & 601 & 67,8 & 9635 & 68,1 & & \\
\hline Total & 886 & 100 & 14149 & 100 & & \\
\hline \multicolumn{5}{|c|}{ Sécurité et développement compromis (SDC) } & 56,68 & $<0,0005$ \\
\hline Non & 264 & 35,4 & 5446 & 49,6 & & \\
\hline Oui & 482 & 64,6 & 5528 & 50,4 & & \\
\hline Total & 746 & 100 & 10974 & 100 & & \\
\hline \multicolumn{5}{|c|}{ Nombre de signalements antérieurs fondés } & 1,02 & $<0,0005$ \\
\hline Aucun & 491 & 53,8 & 10313 & 69,6 & & \\
\hline Un signalement & 265 & 29,1 & 2967 & 20,0 & & \\
\hline Deux ou plus & 156 & 17,1 & 1539 & 10,4 & & \\
\hline Total & 912 & 100 & 14819 & 100 & & \\
\hline \multicolumn{5}{|l|}{ Placements } & 10,94 & $<0,05$ \\
\hline Non & 343 & 71,2 & 4298 & 77,7 & & \\
\hline Oui & 139 & 28,8 & 1230 & 22,3 & & \\
\hline Total & 482 & 100 & 5528 & 100 & & \\
\hline
\end{tabular}




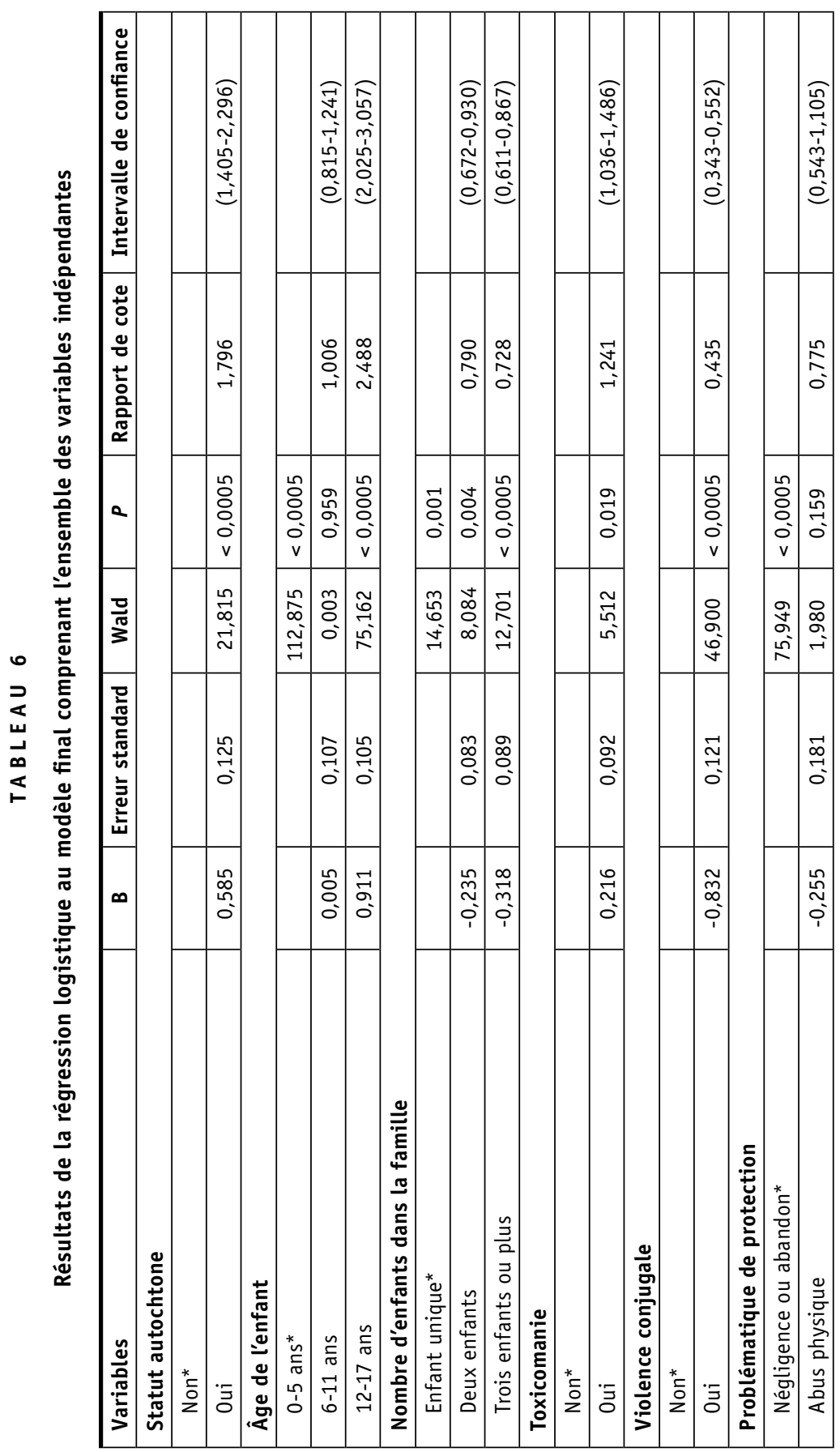




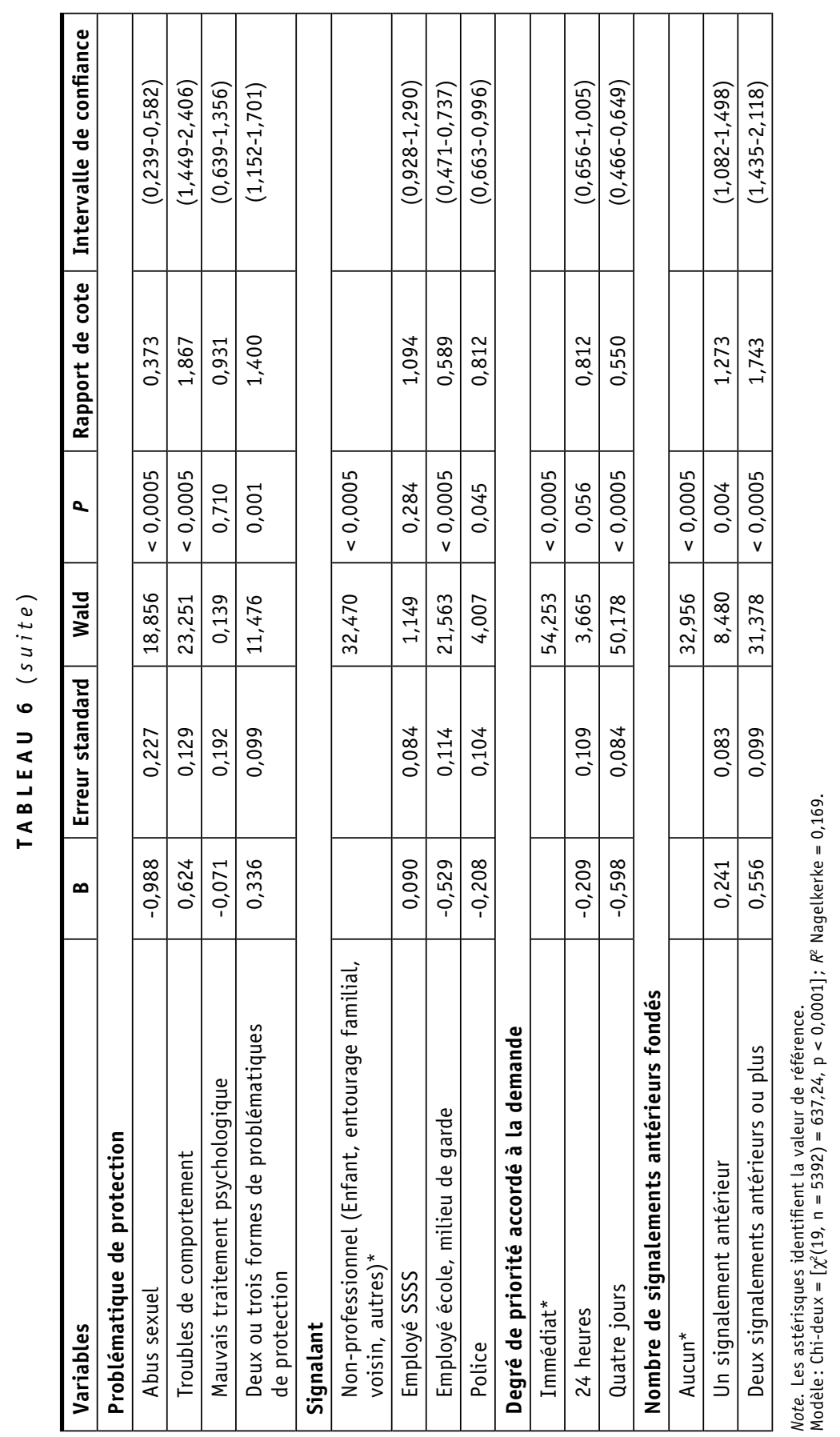


alors que c'est l'inverse pour ceux signalés pour abus sexuel. Les probabilités de placement diffèrent selon de type de signalant. Les probabilités de placement sont moins grandes lorsque le signalement provient de l'école ou du milieu de garde fréquenté par les enfants ou de policiers que d'un signalant non professionnel. Par ailleurs, les résultats montrent que les probabilités de placement sont d'autant plus importantes que les professionnels jugent de la plus grande priorité de faire immédiatement l'évaluation de la situation de l'enfant. Finalement, les enfants qui présentent une situation de récurrence dans les services ont de plus grands risques d'être placés.

\section{Discussion}

\section{Liens entre les résultats et les études antérieures}

Cette recherche établit d'abord la surreprésentation des enfants autochtones québécois à trois étapes de prise de décision en protection de la jeunesse. Cette surreprésentation est cohérente avec celle rapportée dans des études antérieures au Canada et aux États-Unis (Ards et al., 2003; Farris-Manning et Zandstra, 2003 ; Blackstock et al., 2004; Hill, 2007; MacLaurin et al., 2007; Lavergne et al., 2008). Il est préoccupant de réaliser qu'en plus d'être déjà élevée à l'entrée dans le système de protection, la surreprésentation des enfants autochtones augmente à mesure qu'ils progressent dans les services. Dans une étude portant sur des questions similaires auprès des enfants québécois des minorités visibles, Lavergne, Dufour, Sarmiento et Descôteaux (2009) ont constaté que la surreprésentation des enfants noirs aux différentes étapes des services de protection diminue avec la progression dans les services. Comment expliquer que la surreprésentation des enfants autochtones ne suive pas cette même tendance à mesure qu'ils progressent dans la trajectoire de services?

Comparativement à la situation des enfants non autochtones, celle des enfants autochtones se caractérise notamment par le jeune âge des enfants et d'importantes difficultés parentales. En outre, même si elle n'a pas pu être étudiée dans le cadre de la présente recherche, l'extrême pauvreté avec laquelle les parents autochtones doivent conjuguer ne peut être ignorée. Jumelée à la vulnérabilité des enfants et à la présence importante de problèmes sociaux, la difficulté d'accès aux services de première ligne dans les communautés autochtones québécoises pourrait constituer une autre raison: les situations signalées seraient déjà forte- 
ment dégradées, ce qui pourrait expliquer des taux de compromission et de placement plus élevés que pour les enfants non autochtones. Selon la Commission de la santé et des services sociaux des Premières Nations du Québec et du Labrador (CSSSPNQL, 2010), les services de protection de la jeunesse représentent encore trop souvent la porte d'entrée pour recevoir des services sociaux en raison d'un manque de services préventifs de type CLSC et de services communautaires dans ces communautés. Des initiatives telles que le Projet de Développement des Services de première ligne dans les communautés des Premières Nations contribuent à la création d'interventions en amont des difficultés (CSSSPNQL, 2010). En prévenant la dégradation de situations psychosociales propices à l'apparition de mauvais traitements, ce genre d'initiatives pourrait réduire le nombre d'enfants se retrouvant signalés et, à terme, en situation de placement.

Le portrait des enfants autochtones québécois desservis par les services de protection de la jeunesse est assez cohérent avec ceux des études antérieures. À l'instar de Lavergne et al. (2008), les enfants autochtones de l'étude sont plus souvent d'âge préscolaire, signalés par un nonprofessionnel (réseau informel) pour négligence ou en raison d'une situation de cooccurrence des mauvais traitements. Ils ont aussi plus souvent des parents aux prises avec des problèmes personnels, résultats similaires aux études antérieures (Donald et al., 2003; Blackstock et al., 2004; Tourigny et al., 2007; MacLaurin et al., 2008). Les enfants autochtones se voient plus souvent considérés en situation de compromission de leur sécurité ou de leur développement, ont plus fréquemment fait l'objet de signalements antérieurs fondés et sont plus souvent placés, résultats qui font écho à ceux de Blackstock et al. (2004). En outre, tout comme dans l'étude de Tourigny et al. (2007), les enfants autochtones reçoivent plus souvent un degré de priorité immédiat pour l'évaluation de leur situation à la réception des signalements.

La présente recherche visait aussi à identifier les caractéristiques pouvant augmenter les probabilités de placement de l'ensemble des enfants desservis par la protection de la jeunesse au Québec, en vérifiant l'importance du statut autochtone comme prédicteur du placement. Concernant les caractéristiques de l'enfant, tout comme ceux d'études antérieures, les résultats de la présente recherche ont montré que les enfants plus vieux (12-17 ans) avaient significativement plus de probabilités d'être placés que les enfants d'âge préscolaire (Segal et Schwartz, 1985; Kohl et al., 2005; Tourigny et al., 2007). Au sujet des caracté- 
ristiques des signalements, toujours en cohérence avec les résultats de Trocmé et al. (2004), un signalement pour troubles de comportement chez un enfant augmente significativement ses risques de placement. Les risques importants de placement chez les enfants signalés pour négligence sont corroborés par d'autres recherches (Lindsey, 1991; Pauzé et al., 1993). Concernant les caractéristiques des services rendus à l'enfant, un enfant ayant fait l'objet de signalements antérieurs fondés voit ses risques de placement augmenter, ce qui concorde avec les résultats de McConnell, Llewellyn et Ferronato (2006). Le fait d'avoir déjà eu un dossier ouvert en protection de la jeunesse n'est toutefois pas une variable significative quant à sa capacité à prédire le placement dans l'étude de Trocmé et al. (2004).

Enfin, la présente recherche se distingue des précédentes en ce qui a trait à la variable culturelle. Ni Trocmé et al. (2004) ni Fluke et al. (2010) n'ont établi que parmi un ensemble de caractéristiques pouvant contribuer au placement, le statut autochtone constitue un prédicteur. Pourtant, dans la présente étude, le fait d'être autochtone double pratiquement le risque de placement $(\mathrm{RC}=1,8)$, effet qui n'est pas annulé par l'ajout d'autres variables relatives aux signalements, aux services rendus, à l'enfant ou aux parents. Toutefois, certaines variables des études de Trocmé et al. (2004) et de Fluke et al. (2010) n'ont pu être analysées puisqu'elles ne faisaient pas partie de la banque de données utilisée. La présence de ces autres variables (par ex., pauvreté, aspects organisationnels) aurait peut-être diminué le poids du statut autochtone dans la prédiction du placement et augmenté la variance expliquée de la régression. Néanmoins, le résultat de la présente étude fait écho aux autres hypothèses avancées dans les écrits sur les causes possibles de la disproportion, à savoir la présence de biais culturels dans les pratiques des professionnels et le manque d'accès à des services adaptés sur le plan culturel susceptibles de prévenir ou de résoudre les difficultés vécues par les jeunes.

\section{Limites et forces de l'étude}

La présente étude comporte certaines limites. D’abord, les données analysées sont des données de services, recueillies au départ pour des fins administratives et cliniques. Bien que d'importants efforts aient été déployés en vue de rendre ces données utilisables en recherche (Lavergne et al., 2005), certaines limites demeurent. Ainsi, la situation 
socioéconomique des familles n'est pas documentée dans le système clientèle des centres jeunesse, alors qu'elle constitue un facteur de risque important des mauvais traitements (Clément et Dufour, 2009). Autre limite, les données de services documentent peu les difficultés de fonctionnement des enfants. Il est donc impossible de confirmer les résultats d'études antérieures, selon lesquels les enfants autochtones recevant des services de protection n'avaient pas plus, voire moins de difficultés de fonctionnement que les autres enfants (Blackstock et al., 2004; Trocmé et al., 2004; Tourigny et al., 2007).

Cette étude représente malgré cela un apport certain aux connaissances concernant les mauvais traitements envers les enfants autochtones et les services qu'ils reçoivent de la protection de la jeunesse. Elle constitue un des premiers efforts destinés à documenter la situation de ces enfants au Québec et leur surreprésentation. L'excellent taux de participation des centres jeunesse desservant des communautés autochtones $(9 / 11)$ et l'inclusion de tous les enfants signalés pour la période à l'étude permettent enfin d'affirmer que le portrait dressé est juste et fiable.

\section{Implications pour la recherche et la pratique}

Les résultats de la présente étude ont certes permis de comparer les enfants autochtones québécois aux enfants non autochtones, mais les différences pouvant exister entre les enfants autochtones, issus de minorités visibles et blancs restent à étudier. Des études antérieures ayant déploré le placement des enfants autochtones dans des cultures différentes de la leur dans le reste du Canada (Blackstock et Bennett, 2003 ; Farris-Manning et Zandstra, 2003), il serait utile d'étudier cette question en territoire québécois. Il serait en outre intéressant de documenter d'autres facteurs liés à l'étiologie des mauvais traitements, dont la pauvreté, la précarité d'emploi, ainsi que les conditions de vie inférieures aux normes, qui accablent souvent les familles autochtones (Blackstock et al., 2004). Enfin, les données de services en protection de la jeunesse constituent une mine importante d'informations pouvant être associées à d'autres mesures complémentaires (données socioéconomiques, consultations qualitatives des familles autochtones). Pour réellement saisir la réalité des familles autochtones québécoises, l'usage d'un devis de recherche mixte, combinant les forces des approches qualitatives et quantitatives, serait susceptible de dresser un portrait plus réaliste et nuancé de la situation des familles autochtones au Québec. 
Sur le plan de la pratique, la surreprésentation des enfants autochtones dans les services de protection de la jeunesse peut être révélatrice de besoins importants non comblés, pouvant augmenter l'apparition des mauvais traitements. Cette recherche interpelle aussi les lacunes dans les filets de sécurité préventifs, qui, s'ils étaient efficaces, pourraient diminuer le contact de ces familles avec les services de protection. La CSSSPNQL (2010) soutient d'ailleurs que l'accès à des services sociaux pour les Autochtones ne devrait plus passer par les services de protection de la jeunesse, mais plutôt par des ressources similaires aux centres locaux de services communautaires (CLSC), pouvant offrir en amont des services communautaires adaptés culturellement avant que des problèmes trop importants n'apparaissent (CSSSPNQL, 2010).

\section{Conclusion}

Depuis quelques années, des chercheurs tentent de mieux comprendre les difficultés psychosociales vécues dans les communautés autochtones, dans l'objectif de développer des interventions plus sensibles à leur réalité. La présente recherche s'ajoute aux études antérieures pour établir la surreprésentation des enfants autochtones dans les services de protection de l'enfance. Les enfants autochtones du Québec ont proportionnellement plus souvent des conditions de vie difficiles et sont exposés à davantage de facteurs de risque que les enfants non autochtones. D'autres recherches sont nécessaires pour comprendre davantage les vulnérabilités et les forces des enfants autochtones québécois et leurs familles. Les efforts consentis pour développer des stratégies d'intervention préventives et des interventions sensibles à leur réalité culturelle doivent être accentués afin d'influencer positivement la trajectoire de nombreux enfants autochtones et soutenir le plus adéquatement possible les communautés autochtones.

\section{Références}

Ards, S., Myers, S. L., Malkis, A., Sugrue, E., \& Zhou, L. (2003). Racial disproportionality in reported and substantiated child abuse and neglect: An examination of systematic bias. Children and Youth Services Review, 25, 375-392.

Blackstock, C., \& Bennett, M. (2003). National children alliance policy paper on aboriginal children. Ottawa: National Children's Alliance.

Blackstock, C., Trocmé, N., \& Bennett, M. (2004). Child maltreatment investigations among aboriginal and non-aboriginal families in Canada. Violence Against Women, 10, 901-916. 
Bosset, P. (2005). Les mesures législatives de lutte contre la discrimination raciale au Québec: un bilan institutionnel. Nouvelles pratiques sociales, 17 (2), $15-30$.

Carter, V.B. (2009). Predictors of placement into out-of-home care for American Indian/Alaskan Natives compared to non Indians. Children and Youth Services Review, 31, 840-846.

Child Welfare League of America. (2005). Children of color in the child welfare system. Washington: National Data Analysis System.

Clément, M.-E., \& Dufour, S. (2009). La violence à l'égard des enfants en milieu familial. Anjou, Québec: CEC.

Commission de la santé et des services sociaux des Premières Nations du Québec et du Labrador. (2010). Services sociaux: services de première ligne. Consulté en juillet 2010, http://www.cssspnql.com/fr/s-sociaux/s-premligne. htm.

Commission royale sur les peuples autochtones. (1996). À l'aube d'un rapprochement: points saillants du Rapport de la Commission royale sur les peuples autochtones. Consulté 11 octobre 2012, http://www.aadnc-aandc. gc.ca/fra/1100100014597/1100100014637.

Commission sur le racisme systémique dans le système de justice pénale en Ontario. (1995). Rapport de la commission sur le racisme systémique dans le système de justice pénale en Ontario. Toronto: Gouvernement de l'Ontario.

Cotton, M.-È. (2008). Maîtres chez nous? Racisme envers les peuples autochtones au Québec et au Canada. L'autre, cliniques, cultures et société, 9 (3), 361-371.

Donald, K. L., Bradley, L. K., Day, P., Critchley, R., \& Nuccio, K. E. (2003). Comparison between American Indian and non-Indian in out-of-home placements. Families in Society, 84, 267-274.

Eid, P., Turenne, M., \& Magloire, J. (2011). Profilage racial et discrimination systémique des jeunes racisés. Québec: Commission des droits de la personne et des droits de la jeunesse.

Farris-Manning, C., \& Zandstra, M. (2003). Children in care in Canada: A summary of current issues and trends with recommendations for future research. Ottawa: Child Welfare League of Canada.

Fluke, J. D., Chabot, M., Fallon, B., MacLaurin, B., \& Blackstock, C. (2010). Placement decisions and disparities among aboriginal group: An application of the decision making ecology through multi-level analysis. Child Abuse and Neglect, 34, 57-69.

Gaudreault, D. (2009). Amérindiens et Inuits. Portrait des nations autochtones $d u$ Québec. Québec: Gouvernement du Québec.

Gouvernement du Québec. (2006). Vers une politique gouvernementale de lutte contre le racisme et la discrimination. Document de consultation. Québec: Gouvernement du Québec.

Hill, R. B. (2007). An analysis of racial/ethnic disproportionality and disparity at the national, state, and county levels. Washington: Casey-CSSP alliance for racial equity in child welfare. 
Jiwani, Y. (2000). L'enquête sociale générale de 1999 sur la violence conjugale: une analyse. Vancouver, BC: Center for Research on Violence against Women and Children.

Kohl, P. L., Edleson, J. L., English, D. J., \& Barth, R. P. (2005). Domestic violence and pathways into child welfare services: Findings from the National Survey of Child and Adolescent Well-Being. Children and Youth Services Review, 27, 1167-1182.

Lavergne, C., Clément, M.-E., \& Cloutier, S. (2005). PIBE ou la création d'une fenêtre sur des données de recherche dans le domaine de la protection des enfants au Québec. Intervention, 122 (1), 31-41.

Lavergne, C., Dufour, S., Trocmé, N., \& Larrivée, M.-C. (2008). Visible minority, aboriginal, and Caucasian children investigated by Canadian protective services. Child Welfare: Journal of Policy, Practice, and Program, 87, 59-76.

Lavergne, C., Dufour, S., Sarmiento, J. \& Descôteaux, M.-E. (2009). La réponse du système de protection de la jeunesse montréalais aux enfants issus des minorités visibles. Intervention, 131, 233-241.

Lindsey, D. (1991). Factors affecting the foster care placement decision: An analysis of national survey data. American Journal of Orthopsychiatry, 61, 272281.

MacLaurin, B., McCormack, M., Trocmé, N., Blackstock, C., Fallon, B., Knoke, D., Black, T., \& Pitman, L. (2007). Compréhension de la surreprésentation des enfants des Premières Nations dans le système de la protection de l'enfance au Canada: une analyse de l'Étude canadienne sur l'incidence des signalements de cas de violence et de négligence envers les enfants (ECI2003). Les enfants au Canada, hiver, 24-29.

MacLaurin, B., Trocmé, N., Fallon, B., Blackstock, C., Pitman, L., \& McCormack, M. (2008). Comparaison entre les cas d'enfants autochtones et non autochtones ayant fait l'objet d'enquête sur la maltraitance au Canada en 2003. CEPB Information, 66, 1-4.

McConnell, D., Llewellyn, G., \& Ferronato, L. (2006). Context-contingent decision-making in child protection practice. International Journal of Social Welfare, 15, 230-239.

Miller-Perrin, C.L., \& Perrin, R. D. (2007). Child maltreatment: An introduction. Thousand Oaks: SAGE.

Milloy, J. S. (1999). A national crime: The Canadian government and the residential school system, 1879-1986. Winnipeg: University of Manitoba.

Pauzé, R., Béchard, S., \& Toupin, J. (1993). Facteurs de risque associés au placement d'enfants en milieu substitut: recension d'écrits. Apprentissage et Socialisation, 16, 203-212.

Public Health Agency of Canada. (2010). Canadian incidence study of reported child abuse and neglect-2008: Major findings. Ottawa: National Clearinghouse on Family Violence.

Radio-Canada. (2008). Electronic References. Consulté le 11 octobre 2012, http://www.radio-canada.ca/nouvelles/National/2008/06/11/002autochtone-excuses.shtml. 
Salee, D. (2005). Peuples autochtones, racisme et pouvoir d'État en contextes canadien et québécois: éléments pour une ré-analyse: racisme et discrimination: perspectives et enjeux. Nouvelles pratiques sociales, 17 (2), 54-74.

Segal, U., \& Schwartz, S. (1985). Factors affecting placement decisions of children following short-term emergency care. Child Abuse er Neglect, 9, 543-548.

Shaw, T. V., Putnam-Horstein, E., Magruder, J., \& Needell, B. (2008). Measuring racial disparity in Child Welfare. Child Welfare League of America, 87, 23-36.

Sinha, V., Trocmé, N., Fallon, B., MacLaurin, B., Fast, E., \& Thomas Prokop, S. (2011). Kiskisik Awasisak: Remember the Children. Understanding the Overrepresentation of First Nations Children in the Child Welfare System. Ontario, Canada: Assembly of First Nations.

Statistiques Canada (2006a). Profil des enfants, adolescents et adultes autochtones: indicateurs clés tirés de l'Enquête sur les enfants autochtones, 2006 et de l'Enquête auprès des peuples autochtones, 2006. Consulté le 11 octobre 2012, http://www12.statcan.gc.ca/census-recensement/2006/ dp-pd/89-635/index.cfm? lang=fra.

Statistiques Canada (2006b). Portrait de la population canadienne en 2006, selon l'âge et le sexe: résultats. Recensement de la population de 2006 (produit no 97-564-XCB2006002 au catalogue de Statistique Canada). Consulté le 11 octobre 2008, http://www12.statcan.ca/francais/census06/analysis/ agesex/index.cfm.

Tourigny, M., Domond, P., Trocmé, N., Sioui, B., \& Baril, K. (2007). Les mauvais traitements envers les enfants autochtones signalés à la Protection de la jeunesse du Québec: comparaison interculturelle. First People Child and Family Review, 3, 84-102.

Trocmé, N., Fallon, B., MacLaurin, B., Daciuk, J., Felstiner, C., Black, T., Tonmyr, L., Blackstock, C., Barter, K., Turcotte, D., \& Cloutier, R. (2005). Canadian Incidence Study of Reported Child Abuse and Neglect - 2003 : Final report. Ottawa : Minister of Public Works and Government Services Canada.

Trocmé, N., Fallon, B., MacLaurin, B., Sinha, V., Black, T., Fast, E., Felstiner, C., Hélie, S., Turcotte, D., Weightman, P., Douglas, P., \& Holroy, J. (2009). Canadian Incidence Study of Reported Child Abuse and Neglect-2008: Final report. Ottawa: Minister of Public Works and Government Services Canada.

Trocmé, N., Knoke, D., \& Blackstock, C. (2004). Pathways to overrepresentation of Arboriginal children in Canada's welfare system. Social Services Review, 78(49), 577-601.

Trocmé, N., MacLaurin, B., Fallon, B., Daciuk, J., Billingsley, D., Tourigny, M., Mayer, M., Wright, J., Barter, K., Burford, G., Hornick, J., Sullivan, R., \& McKenzie, B. (2001). Canadian Incidence Study of Reported Child Abuse and Neglect: Final report. Ottawa: Minister of Public Works and Government Services Canada. 
ABSTRACT - Research in Canada and elsewhere in the world acknowledges the overrepresentation of aboriginal children in child welfare services. The actual study adds knowledge concerning child maltreatment in the aboriginal children population and the way Quebec child welfare responds to aboriginal children. The overrepresentation of aboriginal children is analyzed at three steps of the child welfare trajectory. Then, the personal and family characteristics of Aboriginal children, of their reports and the services they receive are described. Predictors of out-of-home placement for children receiving child welfare services are finally investigated, with a specific attention to the aboriginal status in that prediction. Results reveal an increase in the overrepresentation of aboriginal children as they progress in the different steps of child protective services trajectory. These children live more often with hard life conditions and have to face more risk factors than other children. The importance of aboriginal status in out-ofhome placement decisions remains substantial, even when many other characteristics increasing out-of-home placement risks are added. Implications for research and practice are discussed.

KEYWORDS - Aboriginal children, child maltreatment, child welfare services, overrepresentation, out-of-home placement.

RESUMEN - Las investigaciones canadienses e internacionales coinciden sobre la sobrerrepresentación de los niños aborígenes en los institutos de menores. La presente investigación aporta informaciones sobre el maltrato y la respuesta de los servicios de protección a la infancia hacia los niños aborígenes quebequenses. El primer objetivo de este estudio consiste en analizar la sobrerrepresentación de dichos niños durante tres etapas de toma de decisiones en lo que respecta a protección al menor. Los niños aborígenes son comparados a los otros niños en lo referente a características personales, familiares, parentales, señalamientos y servicios ofrecidos. También son estudiados y verificados los predictores de internación de los niños destinatarios de los servicios de protección, otorgándole una particular atención en dicha predicción al estatuto de aborigen. Los resultados revelan un aumento de la sobrerrepresentación de los niños aborígenes en las diversas etapas de los servicios de protección. Dichos niños presentan generalmente condiciones de vida difíciles y se ven confrontados a un mayor nivel de riesgo que el resto de los niños. El hecho de ser aborigen es un factor importante en la predicción de su internación, mismo después de agregar un conjunto de características que podrían contribuir a la protección. Las implicaciones tanto para la investigación como para la práctica son tratadas.

PALABRAS CLAVE - Niños aborígenes, maltrato, protección del menor, sobrerrepresentación, internación. 


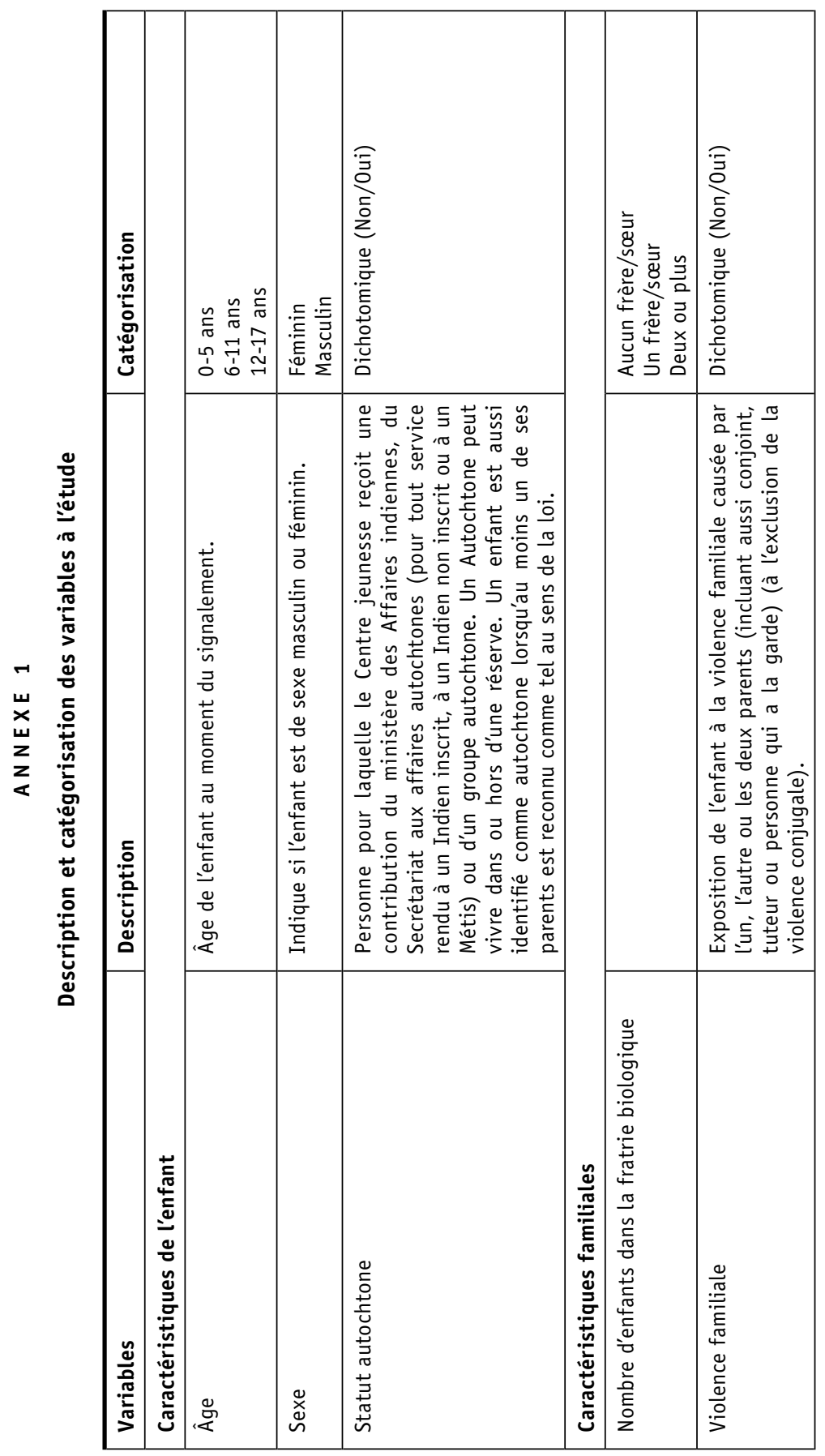




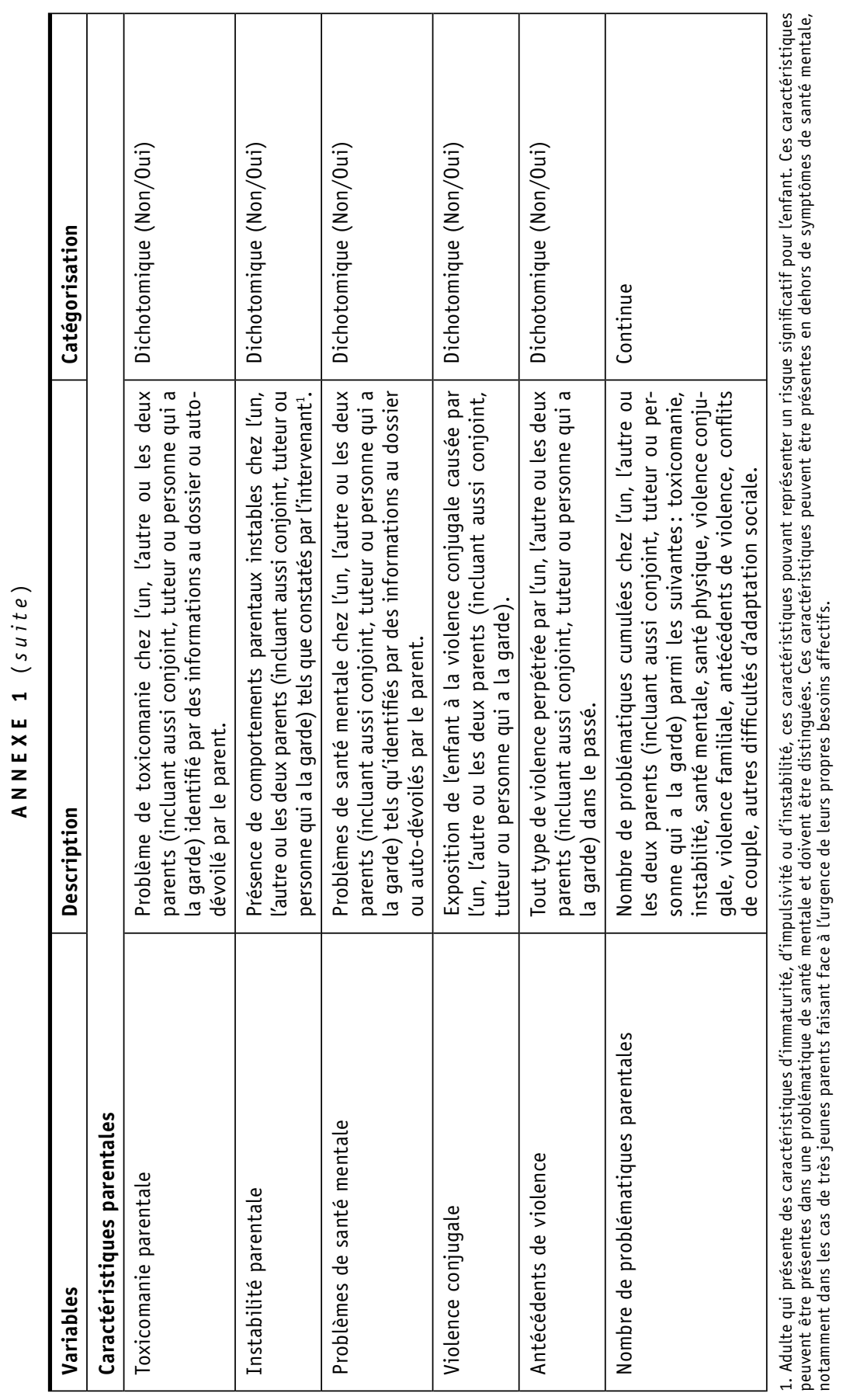




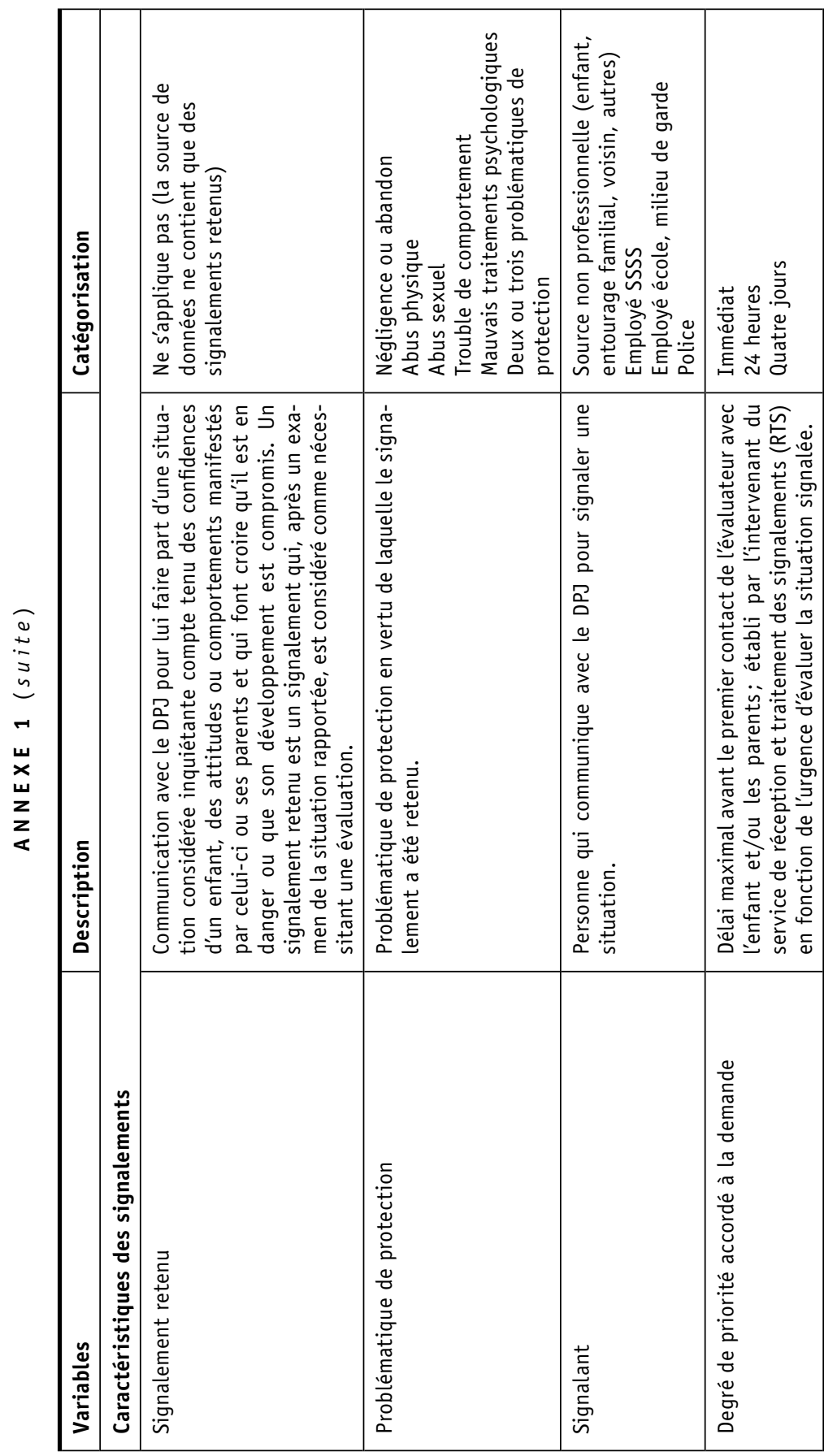




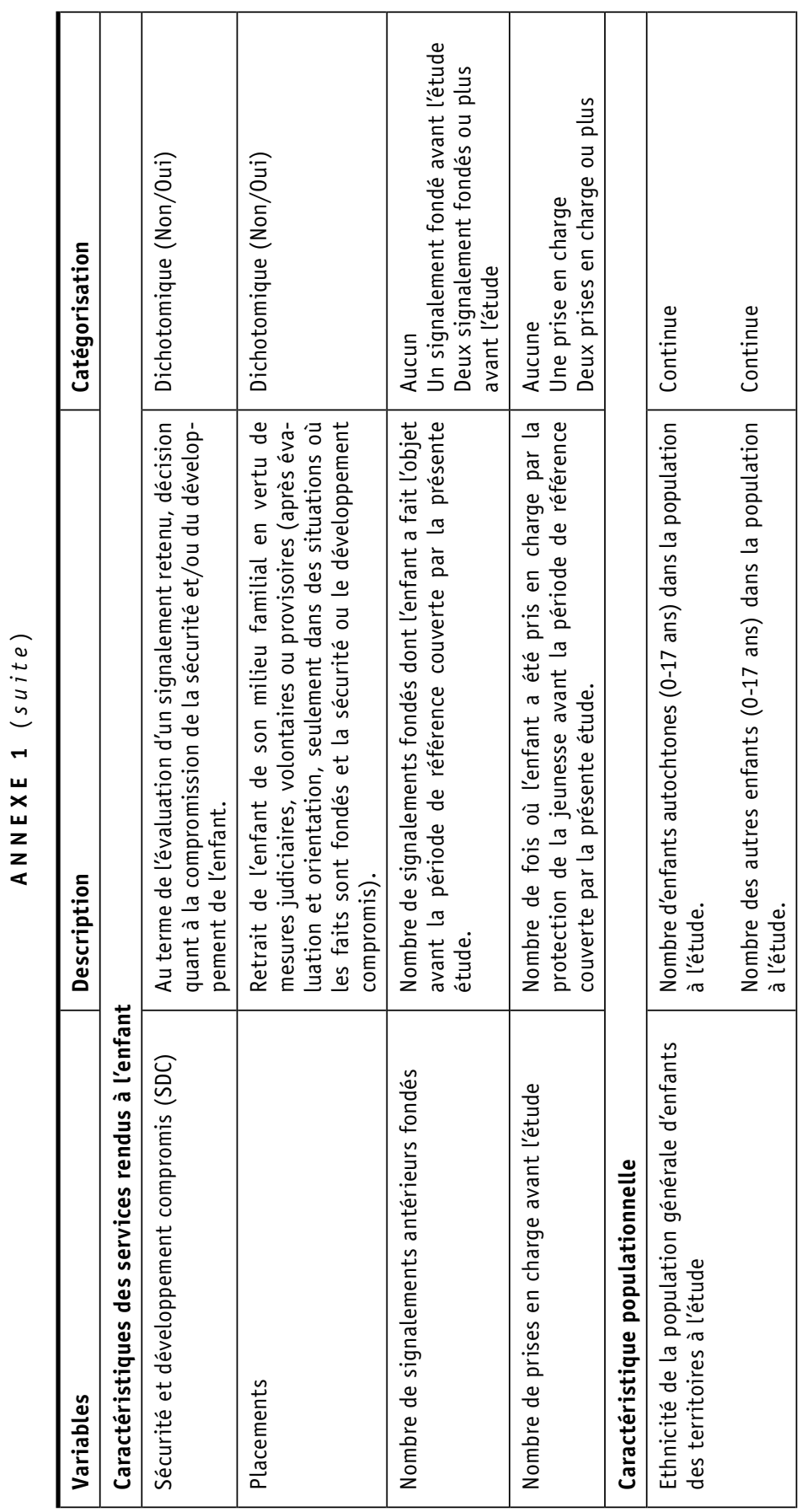

